\title{
Structural and optical properties of PVP-capped nanocrystalline ZnxCd1-xS solid solutions
}

\begin{abstract}
Nanocrystalline ZnxCd1̄̄xS solid solutions were prepared in a microwave-assisted hydrothermal process with gradient distribution of components $(\mathrm{x}=0.1,0.3,0.5,0.7$, and $0.9)$. The growth of the cubic-structured quantum dots was observed for all component stoichiometries with the crystallite size between 4.5 and $5.7 \mathrm{~nm}$. The obvious peak shifts have been found in the XRD patterns and the lattice parameters showed linear variation with $\mathrm{x}$ increasing. The evolution of the optical properties of obtained solid solutions including absorption and photoemission was also monitored in detail. The solid solutions show a considerable shift in the nanoparticle optical absorption edge from 482 to $343 \mathrm{~nm}$ with the increasing of $\mathrm{Zn}$ fraction. The band gaps of the solid solutions were estimated to be between 2.94 and $3.40 \mathrm{eV}$ and the position of conduction band was shifted toward more negative potential with $\mathrm{x}$ increasing. The photoluminescence spectra showed a broad blue-green emission spreading up to $600 \mathrm{~nm}$ with emergence of three dominant peaks belong to sulfur, zinc, and cadmium vacancies.
\end{abstract}

Keyword: Ternary quantum dot; Microwave-assisted hydrothermal; Nanoparticles; Semiconductor 